\title{
Diagnostic Accuracy of Mri Compared to Cet in Patients with Brain Metastases
}

\author{
Firas Salim Daoud Karomy \\ High Diploma in Diagnostic Radiology/ College of Medicine/ University of Baghdad
}

\begin{abstract}
Brain metastasis is one of the most common diagnoses encountered by neurologists, neurosurgeons, radiologists, and oncologists. The aim of this article is to review imaging modalities used in the diagnosis and follow-up of brain metastases. Through the use of various imaging techniques more accurate preoperative diagnosis and more precise intraoperative planning can be made. Post-treatment evaluation can also be refined through the use of these imaging techniques. MRI without and with contrast is the imaging modality of choice in evaluating patients with suspected metastases.28 Head CT is often performed at the initial screening or in an emergency setting to exclude hemorrhage. MRI, however, is superior to CT either without or with contrast in the detection and evaluation of metastases. Metastatic lesions can generally be evaluated with routine contrast MRI studies. Higher dosing of contrast agents, magnetization transfer technique, and higher field strength magnets increase sensitivity.

Typical characteristics of metastases, including multiplicity, location, and signal characteristics, together with clinical history, are often sufficient to suggest the diagnosis of metastatic intracranial disease. In the setting of more complex cases or solitary brain metastases, advanced MRI techniques and PET will aid in reaching a diagnosis.
\end{abstract}

Keywords: neurologists, metastases, preoperative, radiologists, magnetization.

\section{Introduction}

Brain metastasis is one of the most common diagnoses encountered by neurologists, neurosurgeons, radiologists, and oncologists. The aim of this article is to review imaging modalities used in the diagnosis and follow-up of brain metastases. Through the use of various imaging techniques more accurate preoperative diagnosis and more precise intraoperative planning can be made. Post-treatment evaluation can also be refined through the use of these imaging techniques.

Metastatic disease to the brain affects approximately $10 \%$ to $20 \%$ of cancer patients 1 but can be much higher depending on which data series is reviewed (radiologic, surgical, or autopsy). The overall incidence of metastatic brain disease is increasing, which has paralleled the rise in primary lung cancer. Another factor contributing to this increase is the longer survival rates in patients with metastases due to improvements in therapy and better radiologic detection. 2 With respect to autopsy and clinical data, which are more difficult to verify, the incidence of brain metastases is likely to be higher than that of primary brain tumors. ${ }^{3,4,5}$

The general location of metastases in the brain is consistent with blood flow, with approximately $80 \%$ in the cerebral hemispheres, $15 \%$ in the cerebellum, and $5 \%$ in the brain stem. ${ }^{6,7}$ Brain metastases occur secondary to hematogenous spread of neoplastic cells to the brain or tumor emboli.8 Metastases may also involve the calvarium, dura, and leptomeninges, but parenchymal metastases are the most common. The most common sources of metastases are lung (40\% to 50\%), breast $(15 \%$ to $25 \%$ ), and melanoma ( $5 \%$ to $20 \%) .9$ 
Gastrointestinal and renal cell carcinomas are less common but not infrequent. Melanoma is the most likely neoplasm to metastasize to the brain (approximately $50 \%) .{ }^{10,11}$ Although metastases may be multiple (eg, melanoma), solitary metastases are not uncommon, as seen in lung and breast cancers (approximately 50\%).5

\section{General imaging characteristics}

There is no particular location predilection with regard to tumor origin, with a few exceptions. Renal cancer is seen slightly more commonly infratentorially). This may be secondary to retrograde dissemination via Batson plexus, which may account for a slightly higher incidence of posterior fossa metastases from retroperitoneal tumors, such as those of the

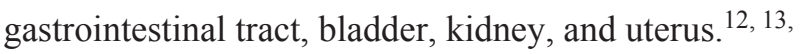

A common general location of parenchymal metastases is the interface of cortical brain and white matter tracts (ie, the gray-white junction), which may be accounted for by the significant change in size of arterioles from cortex to white matter, resulting in lodging of metastases in this location

In addition, metastases are usually surrounded by extensive vasogenic edema. This may be due to tumor capillaries' increased permeability with respect to normal capillaries as well as temporary occlusion as a result of neoplastic cell growth.15, 16 This abnormal vascular permeability allows macromolecules to travel easily into the perivascular and then interstitial spaces. These changes cause increase in pressure on the arterial side of capillary bed and increase in transudation. As a result, peritumoral vasogenic edema, which follows white matter, interdigitating with uninvolved gray matter can be observed on CT but is more obvious on MRI. Cortical metastases, therefore, may not exhibit much surrounding edema because the cortex has a tight interstitium. These metastases may be missed without contrast on both CT and MRI, due to their lack of reactive edema. ${ }^{9}$

Most metastases are hypodense on noncontrast CT but may not be well visualized amidst the surrounding hypodense edema. The pattern of MRI signal intensity of different metastases is not usually helpful in providing a specific diagnosis, but a few general observations have been made. Most metastases demonstrate prolonged relaxation time (high T2 signal).20 Metastases may demonstrate cystic necrosis. Low $\mathrm{T} 1$ and high $\mathrm{T} 2$ signal, however, does not confirm the cystic nature of a neoplasm. Sharp demarcation of lesion with rim enhancement should also be observed. Unlike simple cysts, cystic necrosis demonstrates high signal on fluid-attenuated inversion recovery (FLAIR) sequence (which suppresses signal intensity that is cerebral spinal fluid [CSF]-like). Usually, the T1 rim is not high as expected with an abscess. Necrosis, however, can also shorten relaxation time (low T2 signal).21 This may be secondary to paramagnetics within the tumor (eg, iron or copper) or free radical peroxidation.

Hemorrhage can be associated with metastatic neoplasm and is easily identified acutely on $\mathrm{CT}$ as hyperdensity, which decreases in attenuation after 3 to 4 days, becoming isodense and subsequently hypodense by approximately 7 to 10 days. The paramagnetic effects of blood breakdown products allow intratumoral hemorrhage to be readily identified on MRI for weeks to years after the acute event. Approximately $20 \%$ of metastases are hemorrhagic and a hemorrhagic neoplasm is more likely to be metastatic than primary. The most common hemorrhagic metastases are melanoma, small cell lung cancer, thyroid cancer, choriocarcinoma, and renal cell carcinoma (8)

Fig. 378 year old male with history of bladder cancer and thyroid cancer. (A) Axial T2 and (B) postcontrast T1-weighted sequence demonstrate several left temporal enhancing lesions. (C) Precontrast T1 sequence lesions demonstrate T1 shortening. (D) Lesions demonstrate low gradient recalled echo (GRE) signal compatible with hemorrhagic metastases.

Hemorrhagic metastases, like other hemorrhagic neoplasms, may demonstrate heterogeneous intensity pattern (due to repeated bleeding), an incomplete hemosiderin rim, disproportionate amount of edema for hematoma size, or persistent/increasing edema over 
several weeks. These findings suggest an underlying neoplasm. Additional characteristics that may help to distinguish an uncomplicated parenchymal hematoma from a hemorrhagic metastasis include delayed evolution of hemorrhage within the neoplasm (persistent deoxyhemoglobin-usually seen only for 3 to 5 days) and T1 shortening of intracellular methemoglobin within and not at the periphery of the lesion. Identification of enhancing, non-hemorrhagic tumor component can often seal the diagnosis of hemorrhagic metastasis.(10)

Melanin-containing melanoma metastasis also typically demonstrates $\mathrm{T} 1$ shortening (high signal on T1) and isointense T2 signal due to free radical content of melanin, unlike intracellular methemoglobin, which demonstrates high T1 and very low T2 signal. Confounding this observation is the occurrence of hemorrhage within melanoma metastases. With associated use of a susceptibility sequence, $\mathrm{T} 2 *$, further improved detection of melanoma metastases is possible. At times, melanoma lesions are detected only on $\mathrm{T} 2 *_{-}$ weighted (also known as gradient-echo) sequence. Amelanotic melanoma demonstrates $\mathrm{T} 1$ hypointensity and $\mathrm{T} 2$ hyperintensity. 25

Hypervascularity can be associated with a metastatic lesion, with possible subsequent hemorrhage. Prominent flow voids seen on T2-weighted sequence represent enlarged vessels. The differential diagnosis includes hemangioblastoma, glioblastoma multiforme (GBM), anaplastic oligodendroglioma, and metastatic renal cell carcinoma. Gradient recalled echo (GRE) sequence can confirm both the presence of hemorrhage and prominent vessels.

Low T2 signal (similar to gray matter) is seen in some metastatic neoplasms and may offer a clue to tissue of origin. Mucin-secreting tumors (gastrointestinal and genitourinary adenocarcinomas and at times lung) and hypercellular neoplasms (high nuclear-to-cytoplasmic ratio, small round cell tumors), such as lymphoma (both primary and metastatic), medulloblastoma/ primitive neuroectodermal tumors, pineoblastoma, and neuroblastomas, often demonstrate low $\mathrm{T} 2$ signal.26
These lesions also demonstrate slightly restricted diffusion (high signal on diffusion weighted imaging [DWI] with corresponding reduced apparent diffusion coefficient [ADC] maps), again due to their high cellularity and decreased interstitial space and water Fig. 5.15 Small cell lung cancer, amelanotic melanoma, and some hemorrhagic metastases may also have low T2 signal.

\section{Figure thumbnail gr5}

Fig. 5Metastatic primitive neuroectodermal tumor in a 20-year-old man. (A, B) Isointense T2 signal in a solidly enhancing mass. (C) High DWI signal. (D) Corresponding low ADC map consistent with restricted diffusion.

Calcified metastases are rare and most commonly due to ovarian cancer and osteosarcoma metastases. Calcifications are best seen on CT, because MRI may not provide sufficient discrimination between calcification and hemorrhage. ${ }^{15}$

\section{Contrast-enhanced MRI and CT}

Contrast enhancement is necessary to evaluate for metastatic disease, because nearly all metastases demonstrate enhancement due to lack of blood-brain barrier and lack of blood-tumor barrier in their vascular endothelium. The pattern of enhancement can vary significantly among different metastatic neoplasms but again offers little clue as to the tissue origin. The enhancement pattern may be solid, peripheral, or nodular. The wall characteristics may help distinguish a neoplasm, either metastatic or primary, from a benign process, such as abscess or demyelinating disorder. Irregular thick or nodular enhancement is more commonly seen with malignant neoplasm than with benign conditions, which usually produce smooth thin wall enhancement ${ }^{(11)}$

MRI without and with contrast is the imaging modality of choice in evaluating patients with suspected metastases.28 Head CT is often performed at the initial screening or in an emergency setting to exclude hemorrhage. MRI, however, is superior to $\mathrm{CT}$ either without or with contrast in the detection 
and evaluation of metastases. Approximately $19 \%$ of patients with a solitary metastasis on contrast-enhanced CT have multiple metastases on a contrast-enhanced MRI.29 Improved detection with postcontrast MRI is secondary to superior soft tissue contrast, lack of bone artifact, decreased partial volume averaging, and better enhancement with paramagnetic MRI agents (gadolinium agents) versus CT iodinated. In addition, MRI is better able to identify posterior fossa/infratentorial lesions and leptomeningeal metastatic disease.(13)

Identification of lesions on MRI depends primarily on size of lesion and degree of contrast of lesion to background. The timing and dosing of imaging after gadolinium contrast administration play a major role in visualizing metastases. Delayed imaging postcontrast administration may help in detection of lesions that are $5 \mathrm{~mm}$ or smaller. Waiting 5 to 35 minutes with standard dose improves detection.(12) Higher dosing also detects more lesions and approximately $10 \%$ of such cases alter management. With triple dosing, Yuh and colleagues33 found improved detection of multiple metastases. Delineation of these lesions may be important in developing a therapeutic strategy for a patient. Furthermore, prognostic factors after treatment vary depending on the number of cerebral metastases. ${ }^{(14)}$

Another option for improved visualization of smaller metastases is the use of higher-field MRI scanners. 3T MRI scanners, now readily available in clinical practice, have higher signal-to-noise ratio and longer $\mathrm{T} 1$ relaxation time of gray and white matter, which results in more shortening of $\mathrm{T} 1$ relaxation time by the uptake of contrast. Flow artifacts are more noticeable at higher field strengths and can mimic small metastases but can be readily reduced with saturation pulses. ${ }^{15}$

MR techniques, such as magnetization transfer with single-dose gadolinium diethylenetriamine penta-acetic acid (Gd-DTPA), have also been found as efficient as triple-dose Gd-DTPA. Magnetization transfer allows better visualization of intracranial lesions by preapplying an off-resonance radio frequency pulse, which suppresses the signal of the background tissue.36
Knauth and colleagues36 studied 24 patients with 34 enhancing intracranial lesions. No difference was found in number of lesions detected when comparing standard-dose magnetization transfer T1-weighted images with triple-dose T1-weighted images. Tripledose magnetization transfer T1-weighted studies further increase lesion-to-white matter contrast but do not show additional lesions.

Use of magnetization transfer technique and higherfield magnet scanners, rather than triple dosing, should be considered, particularly in the setting of patients with renal disease, given recent concerns for nephrogenic systemic fibrosis or nephrogenic fibrosing dermopathy, which has been seen in patients with moderate to severe end-stage renal disease after the administration of a high dose of a gadolinium-based contrast agent ${ }^{(15)}$

\section{DWI}

DWI is commonly used in MRI brain studies. DWI reflects the microscopic motion of water in tissue is usually disorganized or Brownian motion. DWI depends primarily on water motion in the extracellular space, with increased signal seen when it is not moving freely. To quantify the degree of water motion, ADC maps are needed, which depict true restriction as dark. The more restricted the motion of water, the lower the ADC value, with darker ADC maps. Conventional DWI imaging is commonly used in the setting of acute stroke where decreased diffusion is present, thought to be secondary to cytotoxic edema and decreased extracellular space.(4)

Necrotic neoplasms can be differentiated from bacterial abscess using DWI and ADC maps. Bacterial abscesses tend to demonstrate increased DWI signal and corresponding decreased ADC values. This reduced diffusion may be due to decreased movement of water molecules by bacteria, inflammatory cells, cellular debris, and macromolecules, such as fibrinogen, found in viscous purulent fluid.39 In addition, bacterial abscesses tend to demonstrate a complete or partial, markedly hypointense T2 rim.40 This finding, together with restricted diffusion, can accurately discriminate cystic or necrotic neoplasm from brain abscess). In 
partly treated abscesses, however, restricted diffusion may not be observed.(1)

Typically, metastatic neoplasms exhibit an elevated $\mathrm{ADC}$, but there is significant overlap with the ADC values of primary neoplasms. In some studiers, necrotic metastases demonstrated some restricted diffusion, as expected with a bacterial abscess.41, 42, 43 Attempts have been made to correlate DWI signal intensity with tumor histology in solid neoplasms. Higher mean ADC values with DWI hypointensity were observed in well differentiated versus poorly differentiated adenocarcinomas (with low signal on T2) and metastases other than adenocarcinoma.44 Small cell carcinoma has also shown hyperintensity on DWI, with low ADC values.(6)

It is the $\mathrm{ADC}$ value in the peritumoral edema that may offer a clue as to the diagnosis of an unknown lesion. Chiang and coworkers46 found that the ADC values of peritumoral edema of metastases and contrastenhancing areas significantly higher than those in highgrade gliomas. This may be due to higher intra- and extracellular water fractions in metastases than in highgrade gliomas. In addition, the significant increase in $\mathrm{ADC}$ in edema surrounding metastases may be due to more fluid production. Primary gliomas, alternatively, have infiltrating cells in the surrounding edema and ADC values might show areas of infiltration. ${ }^{2}$

An extension of DWI is diffusion tensor imaging (DTI), which allows the measurement of direction as well as magnitude of water diffusion in 3-D space. MRI diffusion tensors are generated by applying diffusion gradients symmetrically in at least six radial directions (typically 20 or more). From these, a tensor matrix is converted into differential equations, which yield an eigenvector, reflecting magnitude and direction of diffusion in 3-D space. Mean diffusivity (MD) can be calculated from the eigenvector (magnitude measure) and directionality can be quantified by using fractional anisotropy (FA). FA values range between 0 , perfectly isotropic (spherical), and 1, linear ${ }^{(3)}$
Lower FA values are seen in edema or CSF where diffusion is more spherical and higher FA values are seen in normal white matter tracts where water diffusion is highly directional. The FA value is used in DTI tractography to define the threshold for accepting a voxel as representative of a white matter tract (eg, FA >0.20). A pitfall to remember when interpreting DTI tractography images is that although low FA in white matter may indicate altered diffusion with axon disruption or dysfunction, the presence of edema around otherwise intact axons can produce the false appearance of interrupted or damaged white matter tracts. ${ }^{(2)}$

DTI is used in preoperative planning to identify white matter tracts that need to be preserved during surgery while maximizing resection margins. $\mathrm{Yu}$ and colleagues47 studied 16 patients with brain tumors, both primary and metastatic, and found that diffusion tensor tractography was beneficial in the neurosurgical planning and postoperative assessment.

On conventional MRI, solitary metastases and highgrade gliomas often exhibit similar signal and contrast patterns. In general, both classes of neoplasm exhibit significant surrounding high $\mathrm{T} 2$ signal, or vasogenic edema. Lu and colleagues48 performed DTI on 12 patients with high-grade gliomas and 12 with metastatic lesions. With respect to normal white matter, statistically significant changes are seen in the MD and FA values of the peritumoral T2 signal abnormalities. High-grade neoplasms, however, could not be differentiated from metastatic lesions using peritumoral FA changes, but the peritumoral MD surrounding metastatic lesions was found significantly greater than that surrounding gliomas.48 $\mathrm{MD}$ is likely related to increases in extracellular water, which is greater in vasogenic edema surrounding metastasis. 8 In addition, both increased water content and tumor infiltration can cause more disorganized diffusion and, therefore, decreased FA. So, FA peritumoral changes are similar in high grade gliomas and metastasis - increased water content and tumor cells in high-grade gliomas is equivalent to increased water content alone in metastases. ${ }^{(7)}$ 


\section{Magnetic resonance perfusion}

Perfusion studies were initially performed using nuclear medicine techniques with positron emission tomography (PET) and single-photon emission CT (SPECT) scanners using radioactive tracers. SPECT studies are commonly performed using 99mTchexamethylpropylene amine oxime (99mTc-HMPAO) whereas PET studies for perfusion use Oxygen-15-water $(\mathrm{O}-15 \mathrm{H} 2 \mathrm{O})$. Magnetic resonance (MR) perfusion studies are commonly performed using dynamic susceptibility contrast technique or bolus tracking. A more recent MR perfusion method is arterial spin labeling, which does not require contrast administration. Spins in the inflow arteries are perturbed with radiofrequency pulses and the effect of these perturbed spins on image intensity after they flow into the slice is measured.

Although contrast enhancement demonstrates a breakdown in the blood-brain barrier, it is not measuring underlying local neovascularity. Regional cerebral blood volume maps (rCBV) calculated from perfusion studies have been used to determine microvasculature. A statistically significant correlation has been found between $\mathrm{rCBV}$ and glioma grade, with high-grade gliomas demonstrating higher $\mathrm{rCBV}^{(10)}$

Several recent studies have addressed the use of perfusion imaging in differentiating between highgrade gliomas and metastases. The rCBV values within the high-grade gliomas versus metastases are not significantly different.51, 54 The peritumoral edema of primary high-grade glioma has peritumoral infiltrating cells whereas the peritumoral edema of metastatic lesions does not.

Law and colleagues, in 2002,55 and Chiang and colleagues, in 2004,46 in 2004, noted that rCBV in the peritumoral region is significantly higher in primary gliomas than metastases.54 The peritumoral area surrounding metastases that demonstrates high T2 signal is likely due to vasogenic edema associated with abnormal capillaries of tissue of origin that have increase leakiness. 8 In addition, blood flow in edematous tissue is decreased secondary to local compression of microcirculation by the extravasated fluid.49 In highgrade gliomas, there is breakdown of the blood-blain barrier in the enhancing component. In the peritumoral region, however, the hyperintense $\mathrm{T} 2$ signal likely reflects both vasogenic edema and tumor infiltration, accounting for higher $\mathrm{rCBV}^{\left({ }^{(9)}\right.}$

\section{MR spectroscopy}

MR spectroscopy (MRS) allows the noninvasive evaluation of the chemical makeup of the brain. Proton spectroscopy is what is used in clinical practice. Singlevoxel (where a single volume of tissue is excited) or multivoxel techniques are used for in vivo MRS. The proton spectra produced demonstrate peaks at specific resonant frequencies. Many compounds can be detected but several are more commonly used in clinical practice. Choline is a measure of membrane turnover, because it is involved in membrane synthesis and degradation (resonates at $3.2 \mathrm{ppm}$ ). Choline is usually increased with disorders that cause increase in cellular membrane turnover and in disease states that are associated with a hypercellularity. Creatine is a measure of cellular energy (involved in ATP generation and energy metabolism) and relatively constant in the brain, so it is used as a reference $(3.02 \mathrm{ppm})$. N-acetylaspartate (NAA) is a marker of neuronal viability (both concentration and integrity). NAA is found in mature neurons and neuronal processes (resonates at $2.02 \mathrm{ppm}$ ) and is reduced in disorders that destroy or replace neurons. Lactic acid $(1.33 \mathrm{ppm})$ measures anaerobic metabolism. It is an end product in glycolysis, which builds up when oxidative metabolism is unable to meet energy requirements and is normally very low. Lactic acid is elevated in acute stroke, recent seizure, and high-grade/necrotic neoplasms. Highly cellular lesions that outgrow their blood supply use up oxygen and rely on aneorobic glycolysis, with production of lactate. In addition, certain neoplasms have elevated glycolysis independent of oxidative metabolism. Myo-inositol is a cyclic sugar needed for cell growth and glucose storage. It has a prominent peak at $3.56 \mathrm{ppm}$ that is mainly detected with a short echo time. Located in glia (astrocytes), it indicates gliosis if elevated and is usually decreased in 
high-grade primary brain neoplasms.15 Lipid is seen in cellular breakdown ( 0.8 and $1.5 \mathrm{ppm})$ and is a marker of necrosis, as expected in high-grade malignancy both primary brain tumors and metastases.15(pp112,111) The general, nonspecific pattern for neoplasm is low NAA with high choline. In addition, lactate may be seen as neoplasms disrupt the normal glucose metabolism, with resulting hypoxia. Lipids may also be present in high grade neoplasms, secondary to necrosis. Regions with high lactate also have corresponding elevated CBV on perfusion studies. Elevated CBV is an indirect measure of angiogenesis- one of the features of malignant tumors. In general, ratios of these metabolites are used, such as $\mathrm{NAA} / \mathrm{Cr}$ or $\mathrm{Ch} / \mathrm{NAA}$, and comparison is made of normal brain versus diseased brain. Absolute quantification of brain metabolites is not easy to calculate ${ }^{15}$

Mishra and colleagues56 used MRS and DWI in 52 patients to distinguish ring-enhancing cystic lesions: abscess versus nonabscess. Criteria of for abscess diagnosis of low ADC and presence of lactate and cytosolic amino acids (at $0.9 \mathrm{ppm}$ ) were observed in 25 of 29 cases of abscesses. Criteria for tumor cyst were hypointensity on DWI with higher ADC values and presence of lactate and choline, whereas for benign cyst the criteria were hypointensity on DWI with ADC equal to CSF and presence of lactate and choline as well as amino acids, succinate, and alanine. The sensitivity of DWI for differentiating brain abscess from nonbrain abscess was $72 \%$ and for spectroscopy $96 \%$, with a reported specificity of $100 \%$ for both techniques.

Intratumoral spectral patterns may not be helpful in differentiating primary gliomas from metastases due to significant overlap. ${ }^{15}$ The MR spectra of high-grade glioma compared with brain metastases demonstrate a similar pattern. Two comparative studies performed by the same research group using a long and short echo time $1 \mathrm{H}-\mathrm{MR}$ signal evaluated differentiation of metastases and high-grade astrocytoma. The area under the receiver operating characteristic curve for differentiating these two groups of tumor was poor- $64 \%$ and $59 \%$, respectively. For all other comparisons, for example, GBM versus grade II astrocytomas and metastases versus astrocytoma grade II, the area under the receiver operating characteristic curve was $0.9 .58,59,60$ A study by Law,55 however, using a smaller group of patients, did find that the choline/creatine $(\mathrm{Cho} / \mathrm{Cr})$ ratio was significantly higher in GBM versus metastases. Opstad and colleagues61 recently found a significant difference in lipid to macromolecules peaks, using a short echo time, between these two groups, with higher values for metastases. In an earlier study, Ishimaru and coworkers showed that creatine absence might indicate metastases whereas absence of lipid signal may exclude metastases. 62

More consistent than intratumoral MRS is the finding that peritumoral $\mathrm{Cho} / \mathrm{Cr}$ ratio can help differentiate between high-grade gliomas and metastases.55 Elevated peritumoral $\mathrm{Ch} / \mathrm{Cr}$ ratio was observed in high-grade gliomas but not in metastases. Again this is likely due to peritumoral infiltrating high-grade glioma cells. (11) No significant difference was observed for NAA/ $\mathrm{Cr}$ in peritumoral areas, because there is no neuronal replacement or destruction in either metastases or highgrade glioma.46

Fludeoxyglucose F 18 (18F-FDG) PET has been used in the evaluation of lesions to determine their metabolic activity and has been used in brain tumor imaging. 18F-FDG is actively transported across the blood-brain barrier and into the cell where it is phosphorylated. 18F-FDG uptake is generally high in high-grade tumors. ${ }^{(12)}$

DiChiro and colleagues, 63 in one of the first articles on PET, demonstrated that in all 10 patients with high-grade astrocytomas PET showed a region of high activity and a visible hot spot. This finding was not observed in any of the 13 patients with low-grade gliomas. Not all cases in this study, however, were biopsy proved. Many tumors have high glucose uptake and there is a correlation between uptake and anaplastic transformation. De Witte and colleagues64 showed that the presence of areas of increased FDG uptake in a histologically proved low-grade tumor predicts, in most cases, a deleterious evolution to malignant 
transformation. Others have reported varying degrees of accuracy of PET in differentiating high-grade from low-grade tumors and high-grade tumors from radiation necrosis.

These conflicting reports are due to several limitations of brain PET imaging. Normal brain tissue has a high rate of glucose metabolism. When using PET to image low-grade tumors and even in some high-grade tumors, there may not be sufficient visual difference between normal and tumor glucose uptake to be detectable.65 18F-FDG uptake in neoplasms can vary greatly; high-grade tumors may have uptake that is only similar to or slightly above that in white matter, especially after treatment.66 In distinguishing radiation necrosis from recurrent brain rumors, 18F-FDG PET has provided mixed results depending on methods of analysis. If MRI structural information is available for correlation, however, significant improvement in sensitivity and specificity is noted. In a series of 44 lesions treated with stereotactic radiosurgery, 18F-FDG PET alone had a sensitivity of $65 \%$ in subjects with metastases but reached $86 \%$ when MRI and PET images were coregistered. ${ }^{15}$

In a retrospective study by Kosaka and colleagues, 68 FDG PET was evaluated in the differentiation of CNS lymphoma versus high-grade glioma versus metastatic brain tumor, in 34 patients. By using a standardized uptake value maximum, a cutoff level was obtained to distinguish lymphoma from high-grade gliomas and metastatic brain tumors. When comparing high-grade gliomas to metastatic brain tumors, gliomas tended to have higher standardized uptake values, but significant overlap was found.(14)

Whole-body PET can also be used in suspected brain metastasis to evaluate for the primary lesion.(13)

\section{Conclusion}

Metastatic lesions can generally be evaluated with routine contrast MRI studies. Higher dosing of contrast agents, magnetization transfer technique, and higher field strength magnets increase sensitivity.
Typical characteristics of metastases, including multiplicity, location, and signal characteristics, together with clinical history, are often sufficient to suggest the diagnosis of metastatic intracranial disease. In the setting of more complex cases or solitary brain metastases, advanced MRI techniques and PET will aid in reaching a diagnosis.

Conflict of interest: There is no conflict of interest among the authors.

\section{Funding: Self}

Ethical Clearance: This study is ethically approved by the Institutional ethical Committee.

\section{References}

1. McRobbie DW, Moore EA, Graves MJ, Prince MR. MRI from Picture to Proton. Cambridge university press; 2017 Apr 13.

2. Hoult DI, Bhakar B. NMR signal reception: Virtual photons and coherent spontaneous emission. Concepts in Magnetic Resonance: An Educational Journal. 1997;9(5):277-97.

3. Smith-Bindman R, Miglioretti DL, Johnson E, Lee C, Feigelson HS, Flynn M, Greenlee RT, Kruger RL, Hornbrook MC, Roblin D, Solberg LI. Use of diagnostic imaging studies and associated radiation exposure for patients enrolled in large integrated health care systems, 1996-2010. Jama. 2012 Jun 13;307(22):2400-9.

4. OECD. Publishing. Health at a Glance 2009: OECD Indicators. Organisation for Economic Cooperation and Development; 2009.

5. McRobbie DW, Moore EA, Graves MJ, Prince MR. MRI from Picture to Proton. Cambridge university press; 2017 Apr 13.

6. Sasaki M, Ehara S, Nakasato T, Tamakawa $\mathrm{Y}$, Kuboya Y, Sugisawa M, Sato T. MR of the shoulder with a 0.2-T permanent-magnet unit. AJR. American journal of roentgenology. 1990 Apr;154(4):777-8.

7. Jain S, Madani KS, Swaroop M. Inaugural Women in Medicine Summit: An Evolution of Empowerment in Chicago, Illinois, September 20 and 21, 2019: Event Highlights, Scientific Abstracts, and Dancing with Markers. International Journal of Academic Medicine. 2019 Sep 1;5(3):240. 
8. McDermott R, Lee S, Ten Haken B, Trabesinger AH, Pines A, Clarke J. Microtesla MRI with a superconducting quantum interference device. Proceedings of the National Academy of Sciences. 2004 May 25;101(21):7857-61.

9. Sova A. Effects of Rotational Motion on Dose Coverage in Single Isocenter Multiple Target Stereotactic Radiosurgery (Doctoral dissertation, Creighton University).

10. ^ Ciregia F, Urbani A, Palmisano G. Extracellular vesicles in brain tumors and neurodegenerative diseases. Frontiers in molecular neuroscience. 2017 Aug 31;10:276.

11. ^ "Afshar P, Mohammadi A, Plataniotis KN. Brain tumor type classification via capsule networks. In2018 25th IEEE International Conference on
Image Processing (ICIP) 2018 Oct 7 (pp. 31293133). IEEE.

12. ^ "Mobile B. cari-kerja-angkatan-udara-18. 1hehe. com Layanan Informasi 17 Jam.

13. Doron H, Pukrop T, Erez N. A blazing landscape: neuroinflammation shapes brain metastasis. Cancer research. 2019 Feb 1;79(3):423-36.

14. Sawaya R. Considerations in the diagnosis and management of brain metastases. Oncology (Williston Park, NY). 2001 Sep 1;15(9):1144-54.

15. Maschio M, Aguglia U, Avanzini G, Banfi P, Buttinelli C, Capovilla G, Casazza MM, Colicchio G, Coppola A, Costa C, Dainese F. Management of epilepsy in brain tumors. Neurological Sciences. 2019 Oct;40(10):2217-34. 